\title{
Ganglion cyst of the posterior longitudinal ligament causing lumbar radiculopathy: case report
}

\author{
Hisatoshi Baba ${ }^{1}$, Nobuaki Furusawa ${ }^{1}$, Yasuhisa Maezawa ${ }^{1}$, Kenzo Uchida ${ }^{1}$, Yasuo Kokubo ${ }^{1}$, Shinichi Imura ${ }^{1}$ \\ and Sakon Noriki ${ }^{2}$ \\ ${ }^{1}$ Department of Orthopaedic Surgery; ${ }^{2}$ The First Department of Pathology, Fukui Medical School, Shimoaizuki 23, \\ Matsuoka, Fukui 910-11, Japan
}

\begin{abstract}
We describe a man aged 26 years who presented with a neurological syndrome, which was found on lumbar radioculopathy to be due to a ganglion cyst originating from the posterior longitudinal ligament. Based on MRI findings, a cystic lesion was suspected, a round lesion at L4 level with no connection to the adjacent facet or to the dura matter. During surgery, a liquid-containing cystic lesion was found to originate from the posterior longitudinal ligament at L4 level. The resected cyst was diagnosed histologically as a ganglion cyst. A complete cure was established after surgery and no recurrence was noted at a follow-up 1.7 years postoperatively. A ganglion cyst of the posterior longitudinal ligament should be considered in the differential diagnosis of a cyst in the lumbar region causing neurological complications.
\end{abstract}

Keywords: lumbar spine; posterior longitudinal ligament; ganglion cyst; radiculopathy

\section{Introduction}

Cystic lesions within the lumbosacral spinal canal can cause symptoms and signs such as radiculopathy. These cysts include ganglion ${ }^{1-3}$ and synovial cysts. ${ }^{4-7}$ To our knowledge, no report has previously been described of a ganglion cyst in the posterior longitudinal ligament of the lumbar spine.

In this communication, we describe a young man who presented with severe radiculopathy due to a ganglion cyst of the posterior longitudinal ligament of the lumbar spine.

\section{Case report}

A man aged 26 years was referred to our University Hospital complaining of chronic intractable low back discomfort as well as right leg pain, of approximately 6-month duration. There was no precipitating episode of low back sprain or trauma and he had been healthy previously.

On admission, he could not ambulate because of the right leg pain, which radiated from the middle lower back. His posture was abnormal, with flexion of the lower back and right hip joint and also flexion of the knee. Forced extension of the lumbar spine caused severe radicular pain. Coughing also increased the pain in the lower back and leg regions particularly in the medial aspect of the right lower leg. On physical examination, slight hypaestheisa was noted in the right

Correspondence: Dr H Baba
L4 dermatome. Muscle strength of the quadriceps femoris, tibialis anterior, extensor hallucis longus, was assessed as normal. The straight leg raising test was positive on the right at $50^{\circ}$ and the femoral nerve stretch test was also positive. The deep tendon reflexes were normal, and there was no bladder or bowel dysfunction. Blood tests were normal, and the rheumatoid factor was negative.

Routine plain radiography and computed tomography of the lumbar spine were normal. Magnetic resonance imaging (MRI) showed an intraspinal, extradural, space-occupying lesion at the level of L4 vertebra on the right side. The round lesion was isointense on a T1-weighted image and hyperintense on a T2-weighted sequence (Figure 1a). The intervertebral discs at L3-4 and L4-5 levels were slightly degenerated and bulged posteriorly, but no connection to the round lesion was identified. Gadoliniumdiethylenetriaminepenta-acetic acid administration increased the intensity of the signal in the periphery of the lesion on a T1-weighted image (Figure 1b and c). Increased signal intensity was also present in the area posterior to L4 vertebra, at the level of the posterior longitudinal ligament. The lesion appeared to emanate from the posterior vertebral rim at the level of L4, rather than the facet joint at L3-4 level. Based on these imaging studies, a provisional diagnosis of a cystic lesion, either synovial or arachnoidal, was made.

Surgical exposure of the L3-4 and L4-5 area was achieved through an interlaminar posterior approach on the right side, in order to remove the compressive lesion. No abnormality of the facet joints of L3-4 and 
L4 - 5 was found and the ligamentum flavum appeared normal. A dark-brown cystic mass was detected lying anterior to the L4 nerve root (Figure 2), causing significant impingement on the nerve, but the mass was not attached to the facet joints at L3-4 and L45. The L4 nerve root ran over the cyst and appeared significantly stretched longitudinally and was markedly entrapped as it entered into the intervertebral foramen at L4 vertebra. Although the dura matter and the L4 nerve root were not connected to the cyst, a significant amount of adhesion was present between these structures. A microsurgical technique was thus necessary for careful dissection of the cyst from the anterior aspect of the L4 nerve root. However, we could not extirpate the cyst en bloc because of the large liquid content. Accordingly, we aspirated a considerable amount of the proteinaceous cyst material, thus allowing the medial margin of the lesion to come into view of the operating microscope, after carefully retracting the nerve and dura matter to the left side. The cyst was found to originate from the posterior longitudinal ligament at the level of the L34 intervertebral disc, just proximal and medial to the 'shoulder' of the L4 nerve root. Avoiding excessive retraction of the L4 nerve root, the cyst was removed using a piecemeal resectioning technique under the microscope. Histologically, the resected samples consisted of fibrous tissue with myxoid degeneration. The inner wall of the cyst did not contain synovial lining cells. These findings were consistent with a ganglion cyst originating from the posterior longitudinal ligament (Figure 3).

The patient was ambulatory 3 days after surgery without leg pain, and recovery from the sensory disturbance in the leg was complete within approximately 5 weeks. He was completely well at the latest follow-up visit, 1.7 years after surgery, showing no recurrence of the cyst on MRI studies. a

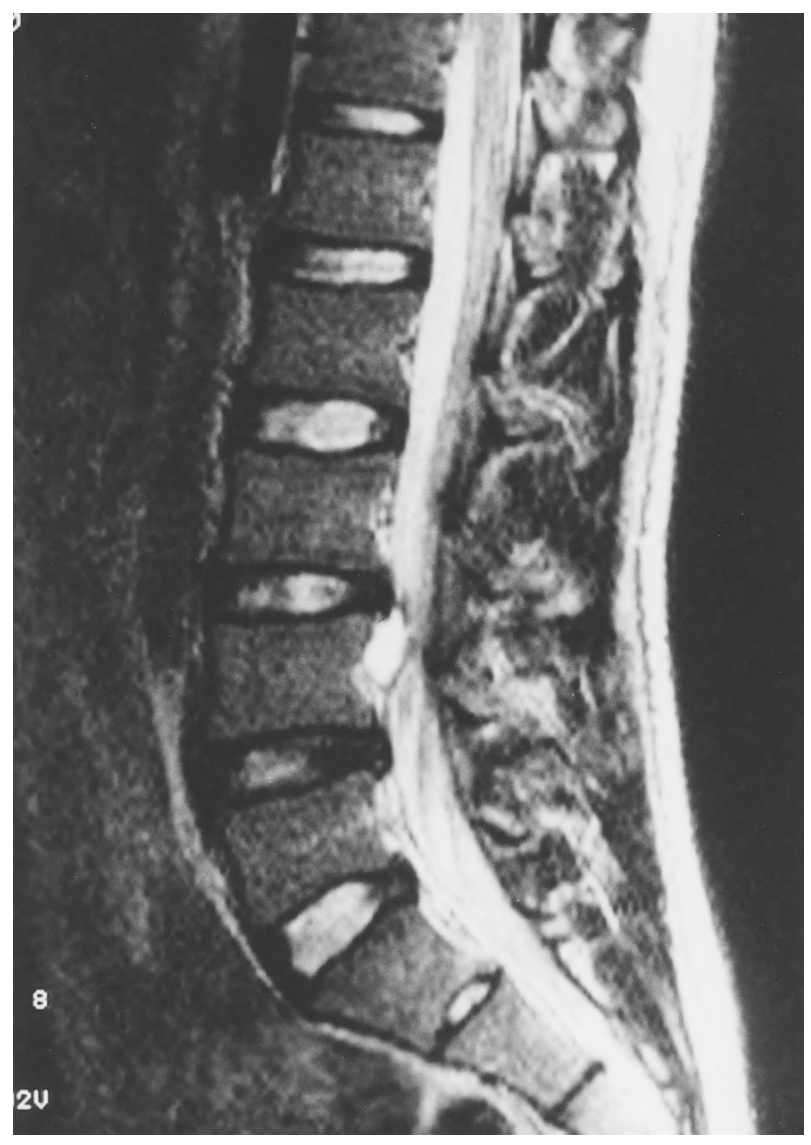

b

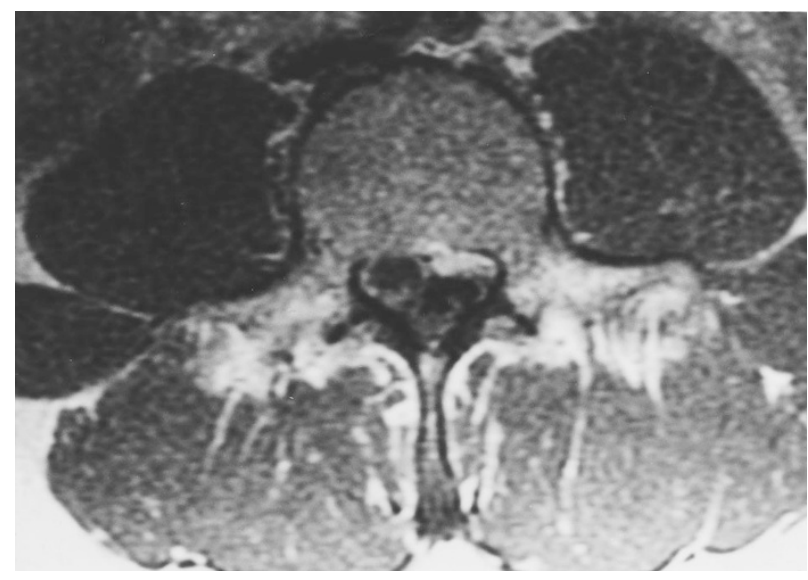

C

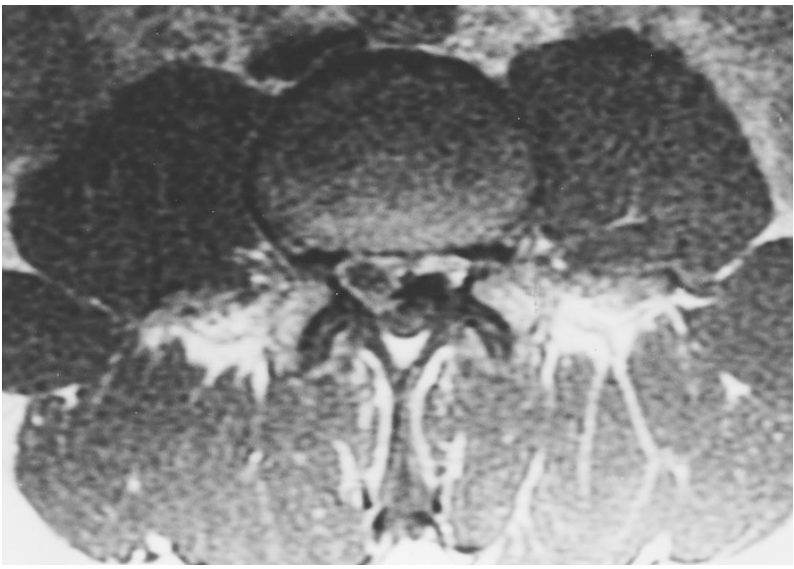

Figure 1 Magnetic resonance imaging findings. (a) The cystic lesion showing a high signal intensity at the level of L4 vertebra (T2-weighted spin echo image; TR, $3500 \mathrm{~ms}$, TE, $20 \mathrm{~ms}$ ). (b) T1-weighted image of the lesion (TR, $450 \mathrm{~ms}, \mathrm{TE}, 20 \mathrm{~ms})$ and (c) gadolinium-diethylenetriaminepenta-acetic acid-enhanced T1-weighted imaging showing increased signal intensity in the margin of the lesion and posterior longitudinal ligament (TR, $450 \mathrm{~ms}$, TE, $20 \mathrm{~ms}$ ) 


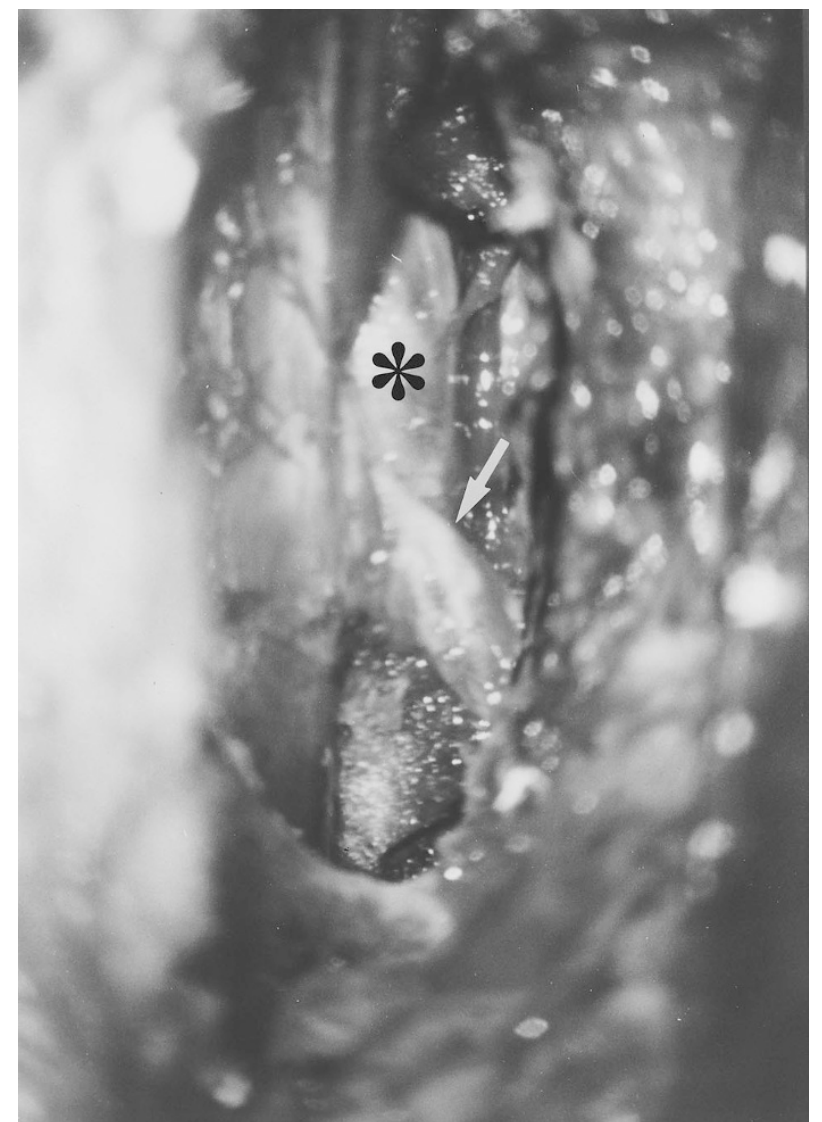

Figure 2 Intraoperative photograph showing the cyst (*) anterior to the right L4 nerve root (arrow)

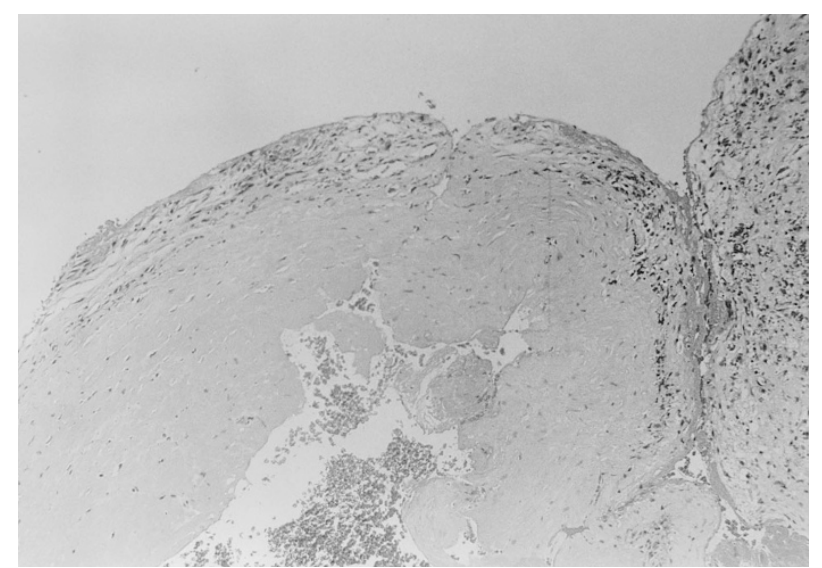

Figure 3 Photomicrograph of the excised cyst showing fibrous tissue in the lesion wall (haematoxylin and eosin, $\times 25$ objective)

\section{Discussion}

A variety of cystic lesions may develop within the lumbar spinal canal potentially compressing the neural structures, causing neurological symptoms. These include arachnoid cyst, epidermoid or dermoid cyst, neurenteric cyst, bronchogenic cyst, perineural cyst (Tarlov), and synovial cyst. Bullough and BoachieAdjei $^{8}$ estimated the prevalance of cysts in the sacral region, extending anteriorly or posteriorly, to be approximately $0.5 \%$ in the general population. Most of these cysts are thought to be of arachnoid origin. A cystic lesion may be found in patients with vertebral arch malformation or spinal dysraphism. In such cases, clear and specific radiological and clinical findings are usually present. On the other hand, several other reports ${ }^{1-3,5-13}$ have described synovial cysts in the lumbar region causing lumbar radiculopathy, and discussed the aetiology and potential complications, including osteoarthritis of the neighbouring facet joints, spondylosis, or degenerative spondylolisthesis. Many authors have suggested that such synovial cysts are connected to the degenerated facet joints, or occasionally to the ligamentum flavum, while some provided evidence that the lesion emanated from the facet joint. ${ }^{8-11,13,14}$ The reports by these authors: Awwad et $a l,{ }^{9}$ Maresca et $a l^{1}$ and Pendleton et $a l^{15}$ are the only ones where there is a discussion of the possibility of ganglion cysts originating from tissues other than the facet joint. To our knowledge, few reports have previously discussed a ganglion cyst with features strongly suggestive of a posterior longitudinal ligament origin.

A ganglion cyst can occur in a variety of tissues and at different locations. The cyst may occur as a result of repeated mechanical stress with subsequent localised histological disruption of the joint capsule, tendon sheath, or cartilage of the knee meniscus. A history of acute or recurrent chronic trauma to the tendon sheath, possibly occupational, may be considered as the cause of a ganglion cyst of the hand, although many cysts remain of unknown aetiology. Recurrent injury with subsequent mucous degeneration of a localised area of the posterior longitudinal ligament of the lumbar spine may be incriminated as the underlying mechanism of cyst formation in our case. In our patient, the intervertebral disc at L3-4 level bulged posteriorly (Figure 1a) and subligamentously, and the condition might have resulted in a small tear of the ligament that subsequently contributed to the development of the ganglion cyst. Prolonged mechanical stimulation of the bulged intervertebral disc might have caused swelling, mucous degeneration, a localised cleavage of the ligament and, finally the formation of a ganglion cyst. However, this hypothesis is not plausible since such a cyst is not present in patients with injury of the posterior longitudinal ligament associated with symptomatic herniation of the lumbar discs. In addition, in many patients with ganglion cysts in the wrist, ankle, or the knee, a concrete evidence of injury of the local connective tissue as the underlying mechanism is not present. Therefore, in the present case, it is likely that the ganglion cyst occurred accidentally in the posterior longitudinal ligament. 
It is generally believed that a ganglion cyst is likely to recur when the cyst is removed only partially. In essence, surgical excision of the ganglion cyst should include the removal of a generous margin at the base. Therefore in our case, prior to excision of the lesion, great care was undertaken to dissect the cyst from the anterior aspect of the L4 nerve root and the dura matter with the help of an operating microscope. Aspiration of the content of the gelatinous cyst facilitated a view of the medial margin of the lesion under the operating microscope. Furthermore, the use of a microsurgical technique allowed a clear identification of the cyst, and excision of the cyst base within the posterior longitudinal ligament at the level of L34 intervertebral disc. The lesion was excised completely with its ligamentous base and, thus, the likelihood of recurrence is minimal.

In conclusion, even though appearing very uncommon, a ganglion cyst should be considered in the differential diagnosis of a mass in the posterior longitudinal ligament causing lumbar radiculopathy. The cyst in our case, which was removed by surgery, consisted of a soft mass compressing the nerve root and the dura matter anteriorly in the lumbar spine.

\section{References}

1 Maresca L, Meland NB, Maresca C, Field EM. Ganglion cyst of the spinal canal: case report. J Neurosurg 1982; 57: 140-142.

2 Martin D, Awwad E, Sundaram M. Lumbar ganglion cyst causing radiculopathy. Orthopaedics 1990; 13: $1177-1183$.
3 Modic MT et al. Contrast-enhanced MR imaging in acute lumbar radiculopathy: a pilot study of the natural history. Radiology 1995; 195: $429-435$.

4 Bjorkengren AG et al. Symptomatic intraspinal synovial cysts: opacification and treatment by percutaneous injection. $A J R$ 1987; 149: 105 - 107

5 Hammer AJ. Synovial cyst - an unusual cause of nerve root compression. S Afr Med J 1988; 73: 44-45.

6 Rosenbaum J, Mojtahedi S, Foust RJ. Synovial cysts in the lumbar spine: MR characteristics. $A J N R$ 1989; 10: S94.

7 Yarde WL et al. Synovial cysts of the lumbar spine: diagnosis, surgical management, and pathogenesis. Report of eight cases. Surg Neurol 1995; 43: 459-465.

8 Bullough PG, Boachie-Adjei O. Developmental and acquired malformations: sacral cysts. Section 3: Disease of the bony elements. In: Atlas of Spinal Diseases. JB Lippincott: Philadelphia 1988, p 126

9 Awward EE, Martin DS, Smith KR Jr, Bucholz RD. MR imaging of lumbar juxtarticular cysts. J Comput Assist Tomogr 1990; 14: $415-417$.

10 Kutz LT et al. Intraspinal synovial cyst causing sciatica. $J$ Bone Joint Surg [Am] 1985; 67: 865-871.

11 Liu SS et al. Synovial cysts of the lumbosacral spine: diagnosis by MR imaging. AJNR 1989; 10: $1239-1242$.

12 Marion PJ, Kahanovitz N. Lumbar-sacral radiculopathy secondary to intraspinal synovial cyst. Arch Phys Med Rehabil 1995; 76: $1011-1013$

13 Mignucci LA, Bell GR. Extradural lesions: lumbar synovial cyst. Chapter 5. Differential diagnosis of sciatica. Section 1. Basic science and diagonsis. In: Herkowitz HN et al. (eds) The Spine. 3rd edn. JB Lippincott: Philadelphia 1992, pp 115116.

14 Tatter SB, Cosgrove GR. Hemorrhage into a synovial cyst causing an acute cauda equina syndrome: case report. $J$ Neurosurg 1994; 81: $449-452$.

15 Pendleton B, Carl B, Pollay M. Spinal extradural benign synovial or ganglion cyst: a case report and review of the literature. Neurosurgery 1983; 13: 322-326. 